\title{
AN ITERATIVE ALGORITHM FOR SYSTEM OF MIXED VARIATIONAL-LIKE INEQUALITIES
}

\author{
Lu-Chuan Ceng And Jen-ChiH YaO
}

Abstract. In this paper we consider a system of mixed variational-like inequalities (for short, SMVLI) involving nondifferentiable terms, and its related system of auxiliary problems in the setting of real Hilbert spaces. An existence theorem for the system of auxiliary problems is established. By exploiting this theorem, an iterative algorithm for SMVLI is constructed. We derive the existence of a unique solution of SMVLI and discuss the convergence analysis of the proposed iterative algorithm. Our results represent the generalization, improvement and development of the previously known results in the literature.

Mathematics subject classification (2010): 49J40, 47J20, 47H06, 49J53, 47H09.

Keywords and phrases: system of mixed variational-like inequalities, system of auxiliary problems, iterative algorithm, convergence analysis.

\section{REFERENCES}

[1] J. P. Aubin, Mathematical Methods of Game Theory and Economics, North-Holland, Amsterdam (1982).

[2] Q. H. AnsARI, S. Schaible, J. C. YAo, System of vector equilibrium problems and its applications, J. Optim. Theory Appl., 107 (3) (2000), 547-557.

[3] Q. H. Ansari, S. Schaible, J. C. YAO, System of generalized vector equilibrium problems with applications, J. Global Optim., 22 (2002), 3-16.

[4] Q. H. AnsARI, J. C. YAO, A fixed point theorem and its applications to the system of variational inequalities, Bull. Austral. Math. Soc., 59 (1999), 433-442.

[5] Q. H. ANSARI, J. C. YAO, System of generalized variational inequalities and their applications, Appl. Anal., 76 (3-4) (2000), 203-217.

[6] S. S. Chang, S. W. XIAng, On the existence of solutions for a class of quasi-bilinear variational inequalities, J. Syst. Sci. Math. Sci., 16 (1996), 136-140.

[7] C. Cohen, F. Chaplais, Nested monotony for variational inequalities over product of spaces and convergence of iterative algorithms, J. Optim. Theory Appl., 59 (1988), 360-390.

[8] M. FERRIS, J. S. PANG, Engineering and economic applications of complementarity problems, SIAM Rev., 39 (1997), 669-713.

[9] R. Glowinski, J. L. Lions, R. Trémolieres, Numerical Analysis of Variational Inequalities, North-Holland, Amsterdam, Netherlands (1981).

[10] N. J. HuAng, C. X. Deng, Auxiliary principle and iterative algorithms for generalized set-valued strongly nonlinear mixed variational-like inequalities, J. Math. Anal. Appl., 256 (2001), 345-359.

[11] G. Kassay,J. Kolumban, System of multi-valued variational inequalities, Publ. Math. Debrecen, 56 (2000), 185-195.

[12] Z. Khan, System of Variational Inequalities and Multiobjective Games, Ph. D. Thesis, Aligarh Muslim University, Aligarh, India (2003).

[13] D. Kinderlehrer, G. Stampacchia, An Introduction to Variational Inequalities and Their Applications, Academic Press, New York (1980).

[14] I. V. Konnov, Combined Relaxation Methods for Variational Inequalities Over Product Sets, Lobachevskii J. Math., 2 (1999), 3-9. 
[15] I. V. Konnov, Combined Relaxation Methods for Decomposable Variational Inequalities, Optim. Methods and Software, 10 (1999), 711-728.

[16] I. V. KonNov, Relatively monotone variational inequalities over product sets, Oper. Res. Lett., 28 (2001), 21-26.

[17] S. Makler-Scheimberg, V. H. Nguyen, J. J. Strodiot, Family of perturbation methods for variational inequalities, J. Optim. Theory Appl., 89 (1996), 423-452.

[18] J. F. NAsh, Equilibrium Point in n-Person Games, Proc. Nat. Acad. Sci. U. S. A., 36 (1950), 48-49.

[19] J. S. PANG, Asymmetric variational inequalities over product of sets: applications and iterative methods, Math. Prog., 31 (1985), 206-219.

[20] L. C. ZenG, S. Schaible, J. C. YAO, Iterative algorithm for mixed variational-like inequalities, J. Optim. Theory Appl., 124 (2005), 725-738.

[21] L. C. ZENG, S. M. GUU, J. C. YAO, Iterative algorithm for completely generalized set-valued strongly nonlinear mixed variational-like inequalities, Comput. Math. Appl., 50, (5-6) (2005), 935945.

[22] A. Nagurney, Network Economics: a Variational Inequality Approach, Kluwer Academic Publishers, Dordrecht (1993).

[23] L. C. ZENG, Iterative algorithm for finding approximate solutions of a class of mixed variational-like inequalities, Acta Math. Appl. Sinicam, English, Ser. 20 (3) (2004), 477-486.

[24] L. C. CENG, J. C. YAO, Approximate proximal algorithms for generalized variational inequalities with pseudomonotone multifunctions, J. Comput. Appl. Math., 213 (2008), 423-438.

[25] L. C. ZENG, N. C. WONG,J. C. YAO, Convergence of hybrid steepest-descent methods for generalized variational inequalities, Acta Math. Sinica, English, Ser. 22 (1) (2006), 1-12.

[26] L. C. ZENG, J. C. YAO, Strong convergence theorem by an extragradient method for fixed point problems and variational inequality problems, Taiwan. J. Math., 10 (5) (2006), 1293-1303.

[27] M. A. Noor, Auxiliary principle for generalized mixed variational-like inequalities, J. Math. Anal. Appl., 215 (1997), 78-85.

[28] M. A. NooR, Iterative schemes for multivalued quasi-variational inclusions, J. Global Optim., 19 (2001), 141-150.

[29] R. U. VERMA, Projection methods, algorithms and a new system of nonlinear variational inequalities, Comput. Math. Appl., 41 (2001), 1025-1031.

[30] Q. H. ANSARI, J. C. YAO, A fixed point theorem and its applications to a system of variational inequalities, Bull. Aust. Math. Soc., 59 (1999), 433-442.

[31] Q. H. ANSARI, J. C. YAO, Iterative schemes for solving mixed variational-like inequalities, J. Optim. Theory. Appl., 108 (2001), 527-541.

[32] S. Schaible, J. C. YAO, L. C. ZENG, On the existence and convergence of approximate solutions for mixed variational-like inequalities, Optimization, 56 (1-2) (2007), 105-114.

[33] L. C. ZENG, L. J. LIN,J. C. YAO, Auxiliary problem method for mixed variational-like inequalities, Taiwan. J. Math., 10 (2) (2006), 515-529. 